\title{
Modelling and Simulation of Wi-Fi Positioning System Deployment for Pedestrian Monitoring
}

\author{
Zhuliang Xu, Kumbesan Sandrasegaran, Cheng-Chung Lin \\ Faculty of Engineering and Information Technology \\ University of Technology Sydney, Centre of Real-time Information Network \\ Zhuliang.Xu@student.uts.edu.au \\ K.Sandrasegaran@uts.edu.au \\ Cheng-Chung.Lin@eng.uts.edu.au
}

\begin{abstract}
Wi-Fi Positioning System (WPS) is a localization technology based on WLAN infrastructure and used the signal strength to implement position determination. With the widely used of Wi-Fi integrated smartphone, WPS has been introduced to monitor pedestrian movement such as movement trend and pedestrian traffic load. The information of pedestrian in a street can be used to improve the services provides to people, for instance, a better bus time schedule and more friendly pavement design. Although there are lots of paper relate to WPS, but very little works has been published to discuss the system deployment. This paper provides modelling and simulation of WPS deployment in a street environment using MATLAB simulation tool. The results of the simulation can be used to optimizing WPS device deployment to achieve better system coverage and efficiency.
\end{abstract}

Keywords — WPS, Deployment, Modelling, Simulation

\section{INTRODUCTION}

Wi-Fi Positioning System (WPS) have drawn the attention of research community in the past few years. It relies on WLAN infrastructure to provide low-cost location service. WPS sniff the packets transmitted by Wi-Fi devices and extract the information of captured packets such as signal strength and captured time. The sniffing device needed for WPS can easy obtain from market: router and Wi-Fi adapter, for instance.

Mobile tracking system is one application of WPS, which is used to monitor the pedestrian movement and density in a street. To detect the available network, the Wi-Fi enabled smartphone which is carried by most of pedestrian traffic points scans the Wi-Fi band intermittently. The typical packet involves in this detecting processing is probe request. Each packet contains a unique device number (MAC address). By deploying the mobile tracking system in an area of interest, the packet which sends by the passed through smartphone can be captured [1]. It is possible, to estimate the location of a smartphone as well as the holding person by using the information of the captured packet such as the MAC address and received signal strength [2, 3].

The mobile tracking system is dividing into two building blocks: sniffing block and administration block.

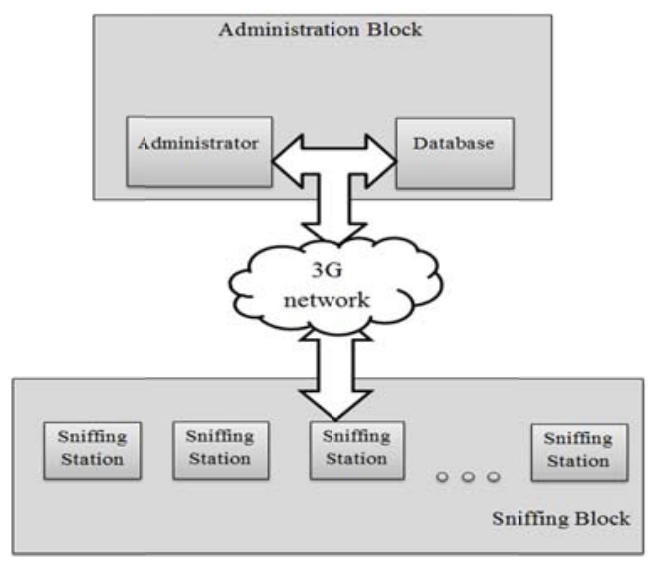

Figure 1: The building block of mobile tracking system

The Figure 1 demonstrates the structure of the building blocks. In the sniffing block, it contains several sniffing stations which are used to capture the Wi-Fi packets. The sniffing station consists of microprocessor, memory, Wi-Fi adapters, antennas and 3G modules. The sniffing station extracts packets related information: hashed MAC address, received signal strength indication (RSSI), captured time and sniffing station ID. Then, the information is written into a file and sends to administration block. In administration block, a database is used for data storage and back up. An administrator can access to the sniffing station remotely via $3 G$ network and obtain the collected data from the database.

Although it is not a new topic to track vehicles and individuals by using WPS, however there is very little work has been published for the system deployment. This paper provides modelling and simulation for WPS deployment in a street environment.

The remainder of this paper is structured as follows. Section 2 describes the system model for mobile tracking. By following, the simulation module is discussed in Section 3.Section 4 contains the simulation environment and results. This paper is concluded in Section 5.

\section{MOBLIE TRACKING SYSTEM MODEL}

In mobile tracking system, the sniffing station passive listens the Wi-Fi channel and captured the transmitted packets. But, Wi-Fi technology has 14 channels, therefore, in order to 
scan all the channels the sniffing station has to apply channel hopping.

In this paper, it is assumed that the street does not have WiFi network and the Wi-Fi enable smartphone broadcast the probe request only. In broadcast procedure, the smartphone sends probe request on one channel and wait at least "MinChannelTime" [4] for response then hop to another channel until all the channels have been scanned. The Figure 2 describes the flowchart of mobile tracking system model.



Figure 2: The mobile tracking system model

\section{SIMULATION MODEL}

A MATLAB simulation tool was developed to model and simulate the sniffing station operation, pedestrian movement and smartphone behaviors. The tool consists of four modules: mobility module, packet generation module, radio propagation module and channel hopping module.

\section{A. Mobility Module}

This module simulates the pedestrian traffic in a street crossing which is shown in Figure 3 and the blue block in the figure is the buildings beside the street. The basic unit for pedestrian traffic is a group and all the individual people within a group have same moving speed and direction. The group can enter or exit this area from 8 ports $(A-H)$ distributed randomly. Some parameters in this module are used to describe the pedestrian traffic: group size, group velocity, traffic load, group reference position and group vector.

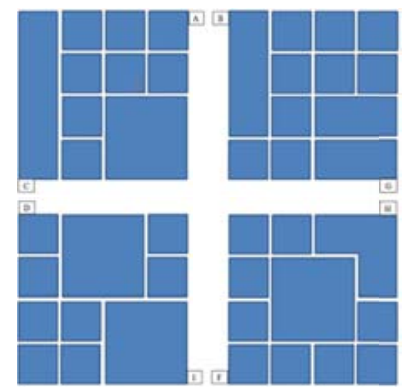

Figure 3: The simulated street crossing

The group size gives the number of members in a group. In a street, most pedestrian group contains 1 and 2 people [5, $6]$. Therefore, in the simulation, the group size for each group is randomly chosen from 1 to 5 with normal distribution which mean is 2 and standard deviation is 3 .
The group velocity presents the pedestrian group walking speed. The Table 1 summarized pedestrian walking speed study in different countries. Based on this study result, the group velocity for each group is randomly chosen with normal distribution which mean is 1.33 and standard deviation is 0.42 .

TABLE I. The pedestrian walking speed [7]

\begin{tabular}{|l|l|l|}
\hline Country & Mean(m/s) & Standard deviation \\
\hline Netherland & 1.14 & 0.22 \\
\hline United State & 1.4 & 0.15 \\
\hline Australia & 1.44 & 0.23 \\
\hline Hong Kong & 1.19 & 0.26 \\
\hline United Kingdom & 1.32 & 1.00 \\
\hline India & 1.46 & 0.63 \\
\hline Average & 1.33 & 0.42 \\
\hline
\end{tabular}

The traffic load is the frequency for a new pedestrian group enters the street crossing. This parameter is used to simulate different pedestrian traffic load in a day such as the peek-time and peek-off time.

The group reference position is used to mark a group location. The new location for group $i\left(\operatorname{loc}_{i}(t+1)\right)$ is determined by using the equation:

$\left.\operatorname{loc}_{i}(t+1)\right)=\operatorname{loc}_{i}(t)+\left(v_{i} \times d i r_{i}\right)$

where $v_{i}$ is the group velocity and $\operatorname{dir}_{i}$ is the moving direction which is a fixed angle $\left(0^{\circ}, 90^{\circ}, 180^{\circ}\right.$ and $\left.270^{\circ}\right)$ depends on the entry port. The moving direction can change $0^{\circ},+90^{\circ}$ or $-90^{\circ}$ randomly when the group meets the street turning.

The group vector $\left(\operatorname{vec}_{j}\right)$ is used to determine the position for each group member $\left(\right.$ position $_{j}$ ). The position for member $j$ in group $i$ can be determined by using the equation:

$\operatorname{position}_{j}(t)=\operatorname{loc}_{i}(t) \times v e c_{j}$

This mobility module is a pass-through method which means the pedestrian group not stays in the simulation area when it reaches the exit port. The tool not updates the information for the left group any more.

\section{B. Packet generation module}

This module generates probe request for the smartphone in each group. As the probe request is very small packet and is transmitted with a fixed data rate $1 \mathrm{Mbps}$. Therefore, this module only considers the packet generates frequency.

A Wi-Fi enabled smartphone has two operation modes: standby mode and active mode. Under active mode, the smartphone is fully running and sends probe request frequent. On other hand, the smartphone sleeps for power saving, when under standby mode. This paper studied 4 major brand smartphones in the markets and the result is presented in Table 2 .

TABLE II. Probe request sending frequency

\begin{tabular}{|c|c|c|c|c|}
\hline Brand & $\begin{array}{c}\text { Operation } \\
\text { System }\end{array}$ & Mode & $\begin{array}{c}\text { Average } \\
\text { value(pack } \\
\text { ets/min) }\end{array}$ & $\begin{array}{l}\text { Standard } \\
\text { Deviation }\end{array}$ \\
\hline \multirow{2}{*}{ HTC } & \multirow{2}{*}{$\begin{array}{c}\text { Android } \\
\text { v2.3 }\end{array}$} & Stanıdby & 0.86 & 0.11 \\
\hline & & Actïve & 3.53 & 0.12 \\
\hline \multirow{2}{*}{ Samsung } & \multirow{2}{*}{$\begin{array}{c}\text { Android } \\
\text { v4.2.1 }\end{array}$} & Stanıdby & 0.60 & 0.40 \\
\hline & & Actïve & 0.73 & 0.64 \\
\hline \multirow{2}{*}{ Apple } & \multirow{2}{*}{ IOS 6.1} & Stanıdby & 0.07 & 0.12 \\
\hline & & Actiive & 1.13 & 1.62 \\
\hline \multirow{2}{*}{ Sony } & \multirow{2}{*}{$\begin{array}{c}\text { Android } \\
\text { v4.1.2 }\end{array}$} & Stanıdby & 0 & 0 \\
\hline & & Actïve & 3.73 & 0.70 \\
\hline
\end{tabular}


By following this table, the simulation tool provides 4 kinds packets sending frequency and randomly allocate to the members in pedestrian group. The allocated packets sending frequency randomly switch between standby mode and active mode by following the mechanism which is shown in Table 3.

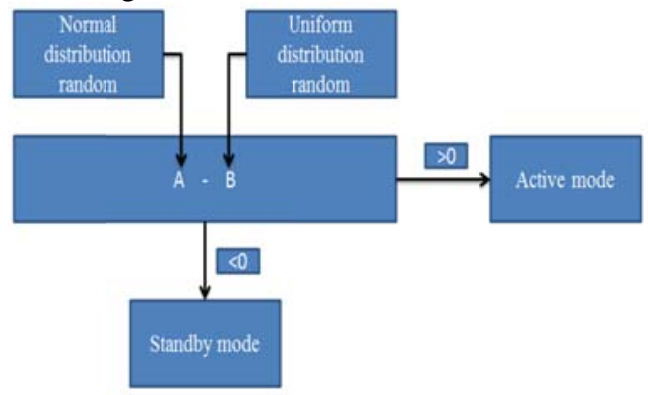

Figure 4: Standby mode and active mode selection

The mean of normal distribution random is 0 , standard deviation is 3 and the mean of uniform distribution random is 2.2 and standard deviation is 0.5 . This method can simulate the smartphone state in a street, which is under standby model in most of time.

\section{Radio propagation Module}

The received signal strength is needed to determine the capture the radio signal is sensible for sniffing station. The radio propagation module uses group velocity and position for each pedestrian group in order to computer the path-loss, shadow and multi-path gain. The log-distance model [8] is used to calculate the path-loss and consists of the following equation:

$$
P L(d B)=P L\left(d_{0}\right)+10 n \log \left(d / d_{0}\right)
$$

where $P L$ is the path-loss, $d_{0}$ is the reference distance which usually is $1 \mathrm{~m}, d$ is the distance between the smartphone and sniffing station and $n$ is the path loss exponent.

By considering the shadowing effect, the Eq.3 becomes to:

$$
P L(d B)=P L\left(d_{0}\right)+10 n \log \left(d / d_{0}\right)+X_{d B}
$$

where $X_{d B}$ is a Gaussian distributed random variable with 0 mean. In terms of $X_{d B}$, it models the path loss variation across all locations from the smartphone due to shadowing.

The path loss exponent in Eq.4 describes the environment condition effect. In the simulation, the $n$ randomly choose from 2.7 to 3.5 when there is line-of-sight between smartphone and sniffing station or randomly choose 3.5 to 5 when the smartphone is blocked by buildings [9].

A simulator developed by Komniakis [10] which using Rayleigh distribution to generate multi-path gain. The multipath gain is related to the pedestrian walking speed and $\mathrm{Wi}-\mathrm{Fi}$ carrier frequency.

Therefore, in this paper, the received signal strength for smartphone hold by group member $j\left(\mathrm{RSS}_{j}\right)$ consists of the following equation:

$$
\operatorname{RSS}_{j}(d B)=T x_{j}(d B)-P L_{j}(d B)-m p a t h_{j}(d B)
$$

where $T x_{j}(d B)$ is the transmitting power for smartphone and mpath $_{j}(d B)$ is the multi-path gain.

\section{Channel hopping module}

This module is used to simulate the channel hopping which is occurred in sniffing station. The hopping time (Th) is the time sniffing station spent to scan one channel. The sniffing station supports three hopping methods which are First-In First-Out (FIFO), Round Robin (RR) and Hop On Last (HOL).

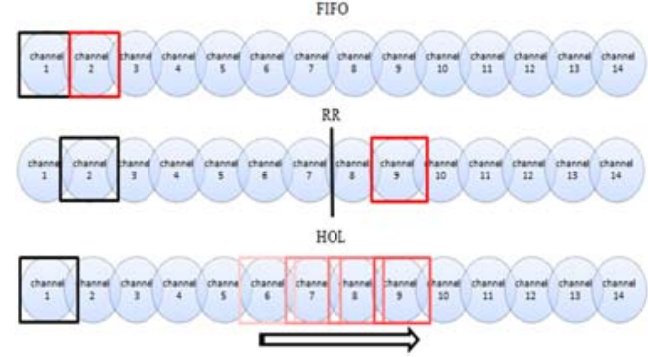

Figure 5: The channel hopping method

The Figure 4 demonstrates the details of the three methods. Specifically, in FIFO method, all the monitoring devices hop simultaneously and following each other. In RR method, every monitoring devise have own hopping section, they scan their own section only. In HOL method, the last monitoring devices hop on the channels and others is fixed to scan one channel only.

\section{IV.SIMULATION RESULT ANALYSIS}

\section{A. Signal station simulation}

The simulation deployment area is the street intersection and the all of the simulation parameters are shown in Table III.

Table III: The mobile tracking system simulation parameters of single station simulation scenario

\begin{tabular}{|cc|}
\hline Parameter & Value \\
\hline Sniffing station sensitivity & $-93 \mathrm{dbm}$ \\
\hline Number of monitoring devices & 2 \\
\hline Traffic load & Fixed number of pedestrian \\
\hline Street length & $100 \mathrm{~m}$ \\
\hline Street width & $30 \mathrm{~m}$ \\
\hline Max_channel_Time & $1 \mathrm{~ms}$ \\
\hline Hopping method & $\mathrm{HOL}$ \\
\hline Selected channels & $1,6,11$ \\
\hline Simulation time & $600000 \mathrm{~ms}$ \\
\hline
\end{tabular}

In the simulation, the factors which can affect packet sniffing are received signal strength and packet generation rate. However, only the signal strength is directly related to the position of the sniffing station.

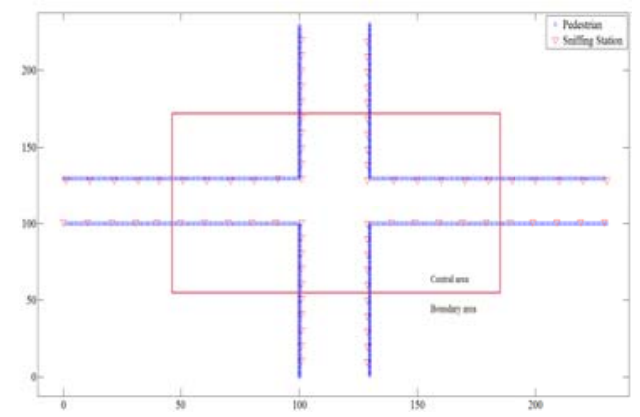

Figure 6: The pedestrian and sniffing stiations distribution of the first scenario

The 800 pedestrians are evenly distributed and their positions are fixed in this simulation scenario. As shown in Figure 6, the simulation area is divided into two parts: central area and boundary area. In the figure, the circle marks represent the positions of pedestrians and pedestrians send packets once. The triangle marks represent the location of sniffing station and 80 locations have been used in the simulation. 
The simulation result is shown in Figure 7 and it can be observed that the sniffing stations captured more packets from the central area than the boundary area. The main reason is that the sniffing stations located at central area and shorter distance to the pedestrians in both horizontal and vertical direction. Specifically, the sniffing station which located at the four highest positions in the Figure 7 has line-of-sight to all pedestrians.

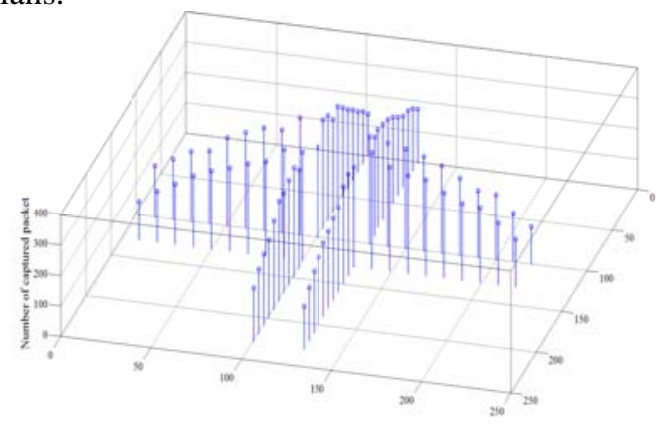

Figure 7: The result of first simulation scenario

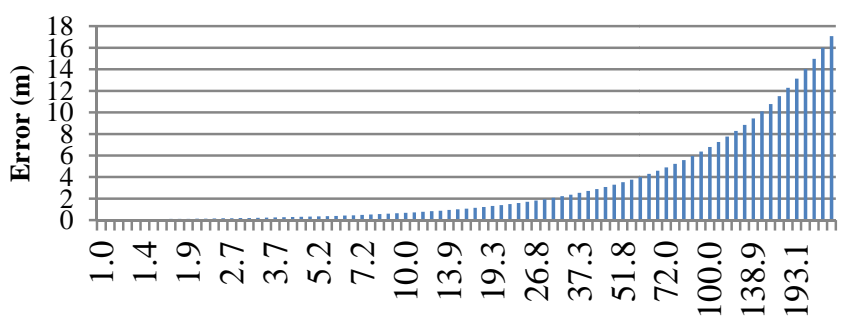

Distance(m)

Figure 8: The localization error at different distance

However, it is hard to achieve optimal localization if all the sniffing stations are deployed within a central area, as the basic requirement of triangulation is that the sniffing stations must well-spaced. Therefore, some sniffing stations have deployed at the boundary area. A simulation was conducted to study the localization accuracy at different distances between sniffing stations and pedestrians. Based on the simulation result which is shown in Figure 8, the localization error for mobile tracking system can be classified into some categories which a shown in Table IV. The option of accurate and good category is more costly as they need more sniffing stations to cover an investigation area. Therefore, the normal and acceptable option is suggested for balance the system cost and localization accuracy.

Table IV: The localization error category

\begin{tabular}{|ccc|}
\hline Category & Error $(\mathbf{m})$ & Distance $(\mathbf{m})$ \\
\hline Accurate & $<1$ & $<14$ \\
\hline Good & $1 \sim 3$ & $<42$ \\
\hline Normal & $3 \sim 5$ & $<72$ \\
\hline Acceptable & $5 \sim 8$ & $<114$ \\
\hline
\end{tabular}

\section{CONCLUSION}

In this paper a simulation tool which was developed based on MATLAB has been introduced. This tool comprises four major models, the mobility model is used to simulate the freewalking pedestrian movement; the packets generation model is used to simulate the probe request broadcasting. The generation rate for the probe request is based on the field test results. Radio propagation model is used to calculate the received signal strength and the channel hopping model is used to simulate the channel scanning, packets capturing and channel hopping function of the sniffing station. In addition, this simulation tool is validated with field test and obtained the similar result.

After conducting the three simulation scenarios of sniffing station deployment, the investigated area (simulation area) is divided into central area and boundary area and the deployment suggestion is given as: a) more sniffing stations are needed to deploy in central area. b) at each direction of the street intersection, at least one sniffing station is needed to deploy in the boundary area.

\section{REFERENCE}

[1] Musa, A. B. M., and Jakob Eriksson. "Tracking unmodified smartphones using wi-fi monitors." Proceedings of the 10th ACM Conference on Embedded Network Sensor Systems. ACM, 2012.

[2] Yeh, Sheng-Cheng, et al. "A s.tudy on outdoor positioning technology using GPS and WiFi networks." Networking, Sensing and Control, 2009. ICNSC'09. International Conference on. IEEE, 2009.

[3] Chung, Wan-Young. "Enhancied RSSI-based real-time user location tracking system for indoor and outdoor environments." Convergence Information Technology, 2007. International Conference on. IEEE, 2007.

[4] Velayos, Héctor, and Gunnar Karlsson. Techniques to reduce IEEE 802.11 b MAC layer handover time. Vol. 3. TRITAIMIT-LCN, 2003.

[5] Yücel, Zeynep, et al. "Deciphering the Crowd: Modeling and Identification of Pedestrian Group Motion." Sensors 13.1 (2013): 875-897.

[6] Ge, Weina, Robert T. Collins, and R. Barry Ruback. "Visionbased analysis of small groups in pedestrian crowds." Pattern Analysis and Machine Intelligence, IEEE Transactions on 34.5 (2012): 1003-1016.

[7] Daamen, Winnie, and Serge P. Hoogendoorn. "Free speed distributions for pedestrian traffic." TRB-Annual Meeting, Washington. 2006.

[8] Sarkar, Tapan K., et al. "A survey of various propagation models for mobile communication." Antennas and Propagation Magazine, IEEE 45.3 (2003): 51-82.

[9] Rappaport, Theodore S. Wireless communications: principles and practice. Vol. 2. New Jersey: Prentice Hall PTR, 1996.

[10] Komninakis, Christos. "A fast and accurate Rayleigh fading simulator." Global Telecommunications Conference, 2003. GLOBECOM'03. IEEE. Vol. 6. IEEE, 2003. 